\title{
Utilization of apnea-hypopnea index as a novel predictive factor for difficult mask ventilation in the Chinese population under general anesthesia
}

\section{Jia-Yi Wang}

Shanghai 9th People's Hospital, Shanghai Jiao Tong University School of Medicine Jing-Jie Li

Shanghai Ninth People's Hospital, Shanghai Jiao Tong University, School of Medicine

\section{Peng-Cheng Zhao}

Shanghai 9th People's Hospital, Shanghai Jiao Tong University School of Medicine

Jia-Li Peng

Shanghai Ninth People's Hospital, Shanghai Jiao Tong University, School of Medicine

\section{Rong Hu}

Shanghai Ninth People's Hospital, Shanghai Jiao Tong University, School of Medicine

\section{Pu Xuan}

Department of quantitative health Sciences, Cleveland Clinic

Hong Jiang ( $\nabla$ dr_jianghongjy@163.com )

Shanghai 9th Peoples Hospital Affiliated to Shanghai Jiaotong University School of Medicine

\section{Research article}

Keywords: Difficult airway management, Difficult mask ventilation, Apnea-hypopnea index

Posted Date: August 19th, 2020

DOl: https://doi.org/10.21203/rs.3.rs-59557/v1

License: (9) This work is licensed under a Creative Commons Attribution 4.0 International License.

Read Full License

Version of Record: A version of this preprint was published at Sleep and Breathing on February 6th, 2021. See the published version at https://doi.org/10.1007/s11325-021-02310-6. 


\section{Abstract}

Background: Difficult Mask Ventilation (DMV) is a potentially life-threatening situation that can arise during anesthesia. Accordingly, the majority of current airway management guidelines include risk assessments for DMV. Although Obstructive Sleep Apnea (OSA) is among the most important risk factors associated with DMV, other measurements such as the Apnea-Hypopnea Index (AHI) may play an important role in determining patient risk.

This study investigated the relationship between DMV and AHI, and determined preoperative risk factors for DMV in Chinese patients.

Methods: A prospective cohort trial enrolled patients scheduled for elective surgery. After obtaining informed consent, patient demographic information was collected, and patients were tested with preoperative polysomnography. Inclusion criteria: Patients $>18$ years of age, American Society of Anesthesiologists Physical Status Classification (ASA) I-III, and planned elective surgery with general anesthesia. Exclusion criteria: malformations of the airway, patients undergoing regional anesthesia, and patients with contraindications to mask ventilation (i.e. planned awake intubation). A logistic regression model was used to analyze the association between AHI and DMV.

Results: A total of 159 patients were analyzed. For both primary and secondary outcomes, the unadjusted and adjusted odds ratio for DMV showed significant increases of $5 \mathrm{AHI}$ units. $\mathrm{AHI}$, age, and the Mallampati classification were found to be independent predictive factors for DMV.

Conclusions $\mathrm{AHI}$ is associated with DMV as a novel independent risk factor in Chinese patients. Along with age and Mallampati classification, AHI should be included in establishing a superior predictive strategy DMV screening.

Trial registration: Chinese Clinical Trial Registry (Registration number \# ChiCTR17013076; Date of Registration on October $\left.22^{\text {nd }}, 2017\right)$.

\section{Background}

One essential aspect of airway management in general anesthesia is to evaluate the effectiveness of mask ventilation for patients[1]. Among all cases, the incidence of difficult mask ventilation (DMV) is between $0.08-15 \%[2-5]$. Difficult ventilation is considered the major contributing factor for morbidity and mortality related to anesthesia[1, 6]. It is important to evaluate the airway and predict the problems prior to induction; the 4th National Audit Project (NAP4) and other major Anesthesia societies recommend a pre-operative assessment for every patient's airway[1, 7-9]. The American Society of Anesthesiologists (ASA) defines DMV as a situation where it is not possible for an unassisted anesthesiologist 1.) to maintain an oxygen saturation $>90 \%$ using $100 \%$ oxygen and positive pressure ventilation; or, 2 .) to prevent or reverse signs of inadequate ventilation[1]. Over the years, a series of studies on the prediction and treatment of DMV (difficult mask ventilation) have been conducted. An age $>55$, body mass index 
$(\mathrm{BMI})>26 \mathrm{~kg} / \mathrm{m}^{2}$, presence of beard, edentulousness, and snoring are five independent factors related to difficult mask ventilation[3]. In addition, neck radiotherapy, neck circumference, male gender, Mallampti grade, and severely limited jaw protrusion are known as risk factors for DMV $[2,4,10]$.

However, most of the above predictors remain unable to fully evaluate DMV risk. On one hand, most clinical predictors are based on European and US populations. To contrast this, risk assessment for DMV in Chinese populations is complicated by the relatively limited number of studies with these geographic and ethnic populations. Moreover, there are discernable differences between Western and Asian populations; fewer bearded patients can be found in the Chinese population. Other differences between the populations include variations in general head and facial structures. Some of these predictors, such as age, BMI, neck circumference, male, and limited jaw protrusion, are closely related to patients who have obstructive sleep apnea (OSA).

It is well-established that a history of OSA is an independent predictor of impossible mask ventilation[3]. Polysomnography (PSG) is the diagnostic standard of OSA[11]. With the advancement of technology for PSG, devices are becoming more portable and easy for patients to wear. One important parameter from PSG is the apnea-hypopnea index (AHI), which is calculated as the sum of all apneas and hypopneas, divided by total hours of sleep time. An AHI of 15 or more events per hour, or five or more events per hour in the presence of symptoms or cardiovascular comorbidities, is diagnosable for OSA[11, 12].

With limited evidence on the predictors in the Chinese population and a close connection between OSA and DMV, we hypothesized that AHI is a predictive factor of DMV in Chinese population. Secondly, we attempted to discover the predictors which are more appropriate for the Chinese population.

\section{Methods}

\section{Ethical statement}

IRB approval by the Ethics Committee (IRB No. 62017-362-T264) was obtained in September of 2017. A study population of 200 patients older than 18 years old underwent elective surgery, with ASA I-III, and general anesthesia over a 12-month period (October 2018-October 2019). Patients with obvious airway malformations, patients undergoing regional anesthesia, and those with contraindication of mask ventilation (i.e. planned awake intubation) were excluded from the study. All patients provided informed consented prior to surgery.

\section{Measurements}

During the preoperative visit, the following information was collected by two anesthesiologists: age, gender, height, weight, BMI, the presence of facial beard, edentulousness, neck circumference, history of snoring, modified Mallampti classification (modified by Samsoon and Young[13], performed with the patient in the sitting position with the head in full extension, tongue out, and with phonation), thyromental distance (measured with the patient in sitting position and head in extension[14]), ability to extend lower 
jaw, alcohol addiction, history of neck radiotherapy, history of DMV and difficult intubation (DI), and past medical \& surgical history.

All patients were scheduled for over-night polysomnography (PSG). All PSGs were performed using a home sleep testing (HST) device (Alice NightOne, Philips, USA). PSG consisted of continuous recording with three sensors ( respiratory effort belt, nasal cannula, and noninvasive pulse oximetry). PSGs were scheduled to last between 6 to 8 hours and terminated following the final waking. All PSG studies were scored by a registered PSG technician. All studies were reviewed and interpreted by a physician boardcertified in sleep medicine. AHI was obtained from the PSG reports.

During induction of anesthesia, a firm pad was placed under the patient's skull and the head was extended by the neck ("sniffing position"). Preoxygenation of each patient lasted 4 minutes by mask (Flexicare, UK; size 3, 4) with $100 \% \mathrm{O}_{2}$. Each patient was routinely monitored during the whole procedure by electrocardiography, noninvasive blood pressure, oxygen saturation $\left(\mathrm{SpO}_{2}\right)$, and end-tidal carbon dioxide tension (Datex-Ohmeda Avance, GE, USA). The same anesthesiologist who obtained information during preoperative assessments also managed the airway, but was blinded to the PSG results. The anesthesiologist graded the mask ventilation as either easy or difficult. In this study, we rated mask ventilation difficulty according to Langeron et al[3] the following: (1) inability for the unassisted anesthesiologist to maintain oxygen saturation as measured by pulse oximetry ( $\mathrm{SpO} 2>92 \%$ ) using $100 \%$ oxygen and positive-pressure mask ventilation; (2) important gas flow leak by the face mask; (3) necessity of increased gas flow to greater than $15 \mathrm{~L} / \mathrm{min}$ and use of oxygen flush valve more than twice; (4) no perceptible chest movement; (5) necessity to perform a two-handed mask ventilation technique; (6) change of operator required[3].

\section{Statistical analysis}

AHI was assumed to be left-skewed and approximately followed a lognormal distribution, with a location parameter of 2 and scale parameters of 1[15]. Based on previous observations, the incidence of difficult group was about $30 \%$. A total of 200 patients would have $90 \%$ power to detect a clinically important odds ratio of 1.25 associated with a 5 -unit increase in $\mathrm{AHI}$.

We summarized the baseline characteristics using standard statistics. Continuous variables with normal distribution were reported as mean \pm standard deviation. Non-normal continuous variables were reported as median (interquartile range). Categorical variables were reported as frequency (percentage).

A logistic regression model was used for the primary analysis to assess the association between $\mathrm{AHI}$ and DMV, without adjusting other variables. Crude odds ratio and a $95 \%$ confidence interval was reported. A pvalue $<0.05$ was considered significant.

The correlation between $\mathrm{AHI}$ and other variables, including age, gender, BMI, neck circumference, modified Mallampti classification, thyromental distance, ability to extend lower jaw, and alcoholism was also explored. Pair-wise Pearson correlation coefficients were reported. Variables that had high correlation 
(rho >0.5) with $\mathrm{AHI}$ were excluded from the multiple logistic regression. Subsequently, a multivariable logistic regression model was used to assess the association of all potential predictors to DMV. Variables with a p-value $<0.05$ were established as independent predictors. The adjusted association between AHI and DMV was explored using multiple logistic regression adjusting for age, gender, BMI, bearded, edentulousness, Mallampti classification, thyromental distance, ability to extend lower jaw, and alcoholism. The adjusted odds ratio and $95 \%$ confidence intervals were reported.

All statistical analysis was conducted using the Statistical Analysis System (SAS) statistical software package (version 9.04.01 SAS Institute Inc, Cary, NC, USA.)

\section{Results}

In this prospective cohort study, a total of 200 cases initially met our inclusion criteria. After excluding cases with missing $\mathrm{AHI}$ and inability to do mask ventilation, 159 cases were included in the final analysis (Figure 1). Patients were aged 18 years or older who underwent elective surgery, with ASA I-III, and general anesthesia. Perioperative baseline characteristics and demographics are shown in Table 1. The median AHI was $10.9[0.0,95]$. The histogram of $\mathrm{AHI}$ is shown in Figure 2. The primary outcome showed that the unadjusted odds ratio (OR) of DMV related with a 5 unit increase of $A H I$ was $1.50[1.29,1.76], P<0.001$. For the secondary outcome, gender, Mallampti classification, thyromental distance, ability to extend lower jaw, and alcoholism were all put in the multivariable logistic regression model. It showed that AHI, age and Mallampati classification were independent factors of DMV. Among these, AHI was highly related to DMV (adjusted OR of DMV with a 5 unit increase of $\mathrm{AHI}, 1.28$ 95\% $\mathrm{Cl}$ 1.08-1.52). Neck circumference was found to correlate with $\mathrm{AHI}$ (correlation coefficient of 0.57 ), and was therefore excluded from the model. In the multivariable analysis, $\mathrm{AHI}$, age, and Mallampti classification continued to maintain their statistical significance $(P<0.05)$, as independent factors (Table 2). Meanwhile, AHI was related to BMI $(P<0.0001)$, age $(P=0.0282)$, gender $(P<0.0001)$, neck circumference $(P<0.0001)$, alcoholism $(P=0.0495)$, thyromental distance $(P=0.0022)$, and Mallampati classification $(P=0.0003)$. The correlation between $A H I$ and other variables is shown in Figure 3. Due to the lack of statistical desirable sample size of patients with beards $(n=1)$ and edentulousness $(n=1)$ in the study population, these two factors were not assessed in the final analysis. 
Table 1. Patients' perioperative demographic and apnoea-hypopnoea Index

\begin{tabular}{|c|c|c|c|c|}
\hline \multirow[b]{2}{*}{ Factor } & \multirow[b]{2}{*}{$\begin{array}{c}\text { Total } \\
(\mathrm{N}=159)\end{array}$} & \multirow{2}{*}{$\begin{array}{c}\begin{array}{c}\text { Easy Ventilation } \\
(\mathrm{N}=103)\end{array} \\
\text { Statistics }\end{array}$} & \multirow{2}{*}{$\begin{array}{l}\begin{array}{l}\text { Difficult Ventilation } \\
(\mathrm{N}=56)\end{array} \\
\\
\end{array}$} & \multirow[b]{2}{*}{ p-value Stddiff } \\
\hline & & & & \\
\hline Age (years) & $38.0 \pm 12.8$ & $34.4 \pm 12.7$ & $44.5 \pm 10.2$ & $<0.001^{a} 0.876$ \\
\hline BMI $\left(\mathrm{kg} / \mathrm{m}^{2}\right)$ & $25.2 \pm 4.7$ & $23.9 \pm 4.3$ & $27.7 \pm 4.3$ & $<0.001^{a} 0.872$ \\
\hline male & $82(51.6)$ & $40(38.8)$ & $42(75.0)$ & $<0.001^{c} 0.784$ \\
\hline $\begin{array}{l}\text { Thyromental distance } \\
\text { (cm) }\end{array}$ & $6.0 \pm 1.2$ & $5.9 \pm 1.2$ & $6.3 \pm 1.10$ & $0.023^{a} 0.387$ \\
\hline $\begin{array}{l}\text { neck_circumference } \\
\text { (cm) }\end{array}$ & $37.0 \pm 4.4$ & $35.5 \pm 3.5$ & $39.8 \pm 4.4$ & $<0.001^{a} 1.073$ \\
\hline $\mathrm{AHI}(/ \mathrm{h})$ & $\begin{array}{c}10.1[3.8 \\
21.2]\end{array}$ & $6.3[2.4,13.3]$ & $20.7[11.2,37.3]$ & $<0.001^{b} 1.312$ \\
\hline bearded & $1(0.63)$ & $1(0.97)$ & $0(0.0)$ & $0.99^{\mathrm{d}}-0.140$ \\
\hline edentulous & $1(0.63)$ & $1(0.97)$ & $0(0.0)$ & $0.99^{\mathrm{d}}-0.140$ \\
\hline alcoholism & $28(17.6)$ & $9(8.7)$ & $19(33.9)$ & $<0.001^{c} 0.646$ \\
\hline radiotherapy & $0(0.0)$ & $0(0.0)$ & $0(0.0)$ & \\
\hline snoring history & $120(75.5)$ & $67(65.0)$ & $53(94.6)$ & $<0.001^{c} 0.794$ \\
\hline limited_jaw_protrusion & & & & $0.14^{\mathrm{d}} \quad 0.335$ \\
\hline . 1 & $144(90.6)$ & $90(87.4)$ & $54(96.4)$ & \\
\hline . 2 & $14(8.8)$ & $12(11.7)$ & $2(3.6)$ & \\
\hline . 3 & $1(0.63)$ & $1(0.97)$ & $0(0.0)$ & \\
\hline Mallampati & & & & $<0.001^{c} 0.789$ \\
\hline . 1 & $77(48.4)$ & $62(60.2)$ & $15(26.8)$ & \\
\hline . 2 & $47(29.6)$ & $28(27.2)$ & $19(33.9)$ & \\
\hline . 3 & $24(15.1)$ & $8(7.8)$ & $16(28.6)$ & \\
\hline $\begin{array}{r}4 \\
\end{array}$ & $11(6.9)$ & $5(4.9)$ & $6(10.7)$ & \\
\hline
\end{tabular}

Statistics presented as Mean \pm SD, Median [P25, P75], Median (min, max) or N (column \%).

p-values: $a=A N O V A, b=$ Kruskal-Wallis test, $c=$ Pearson's chi-square test, $d=$ Fisher's Exact test.

Table 2. Multivariable logistic regression model 


\begin{tabular}{lll}
\hline & OR $(95 \% \mathrm{CI})$ & P-value \\
\hline AHI $(/ \mathrm{h})$ & $1.05[1.02,1.09]$ & $0.0043^{*}$ \\
Age $($ years $)$ & $1.05[1.01,1.09]$ & $0.0075^{*}$ \\
\hline Male & $2.57[0.94,7.06]$ & 0.0664 \\
\hline BMI $\left(\mathrm{kg} / \mathrm{m}^{2}\right)$ & $1.12[0.99,1.26]$ & 0.0830 \\
\hline Mallampti classification & $1.76[1.04,2.96]$ & $0.0341^{*}$ \\
\hline Thyromental distance $(\mathrm{cm})$ & $0.99[0.66,1.50]$ & 0.9667 \\
\hline Limited jaw protrusion & $0.18[0.02,1.52]$ & 0.1164 \\
\hline Alcoholism & $2.16[0.68,6.89]$ & 0.1921 \\
\hline
\end{tabular}

*AHI, age, and Mallampti classification were statistical significance $(\mathrm{P}<0.05)$ factors

\section{Discussion}

This study was designed to determine the association between $\mathrm{AHI}$ and. We found that with the 5-unit increase of $\mathrm{AHI}$, the incidence of DMV was significantly higher. The secondary outcome showed that $\mathrm{AHI}$, age, and Mallampati classification were independent factors of DMV. Indeed, AHI was shown to be highly related to DMV. Normally, the most widely used definition of DMV was by Han et al[16], which was described as "inadequate, unstable, or requires two providers" with or without muscle relaxant. Impossible mask ventilation was defined as the inability to mask ventilate with or without muscle relaxant. In this study, we rated mask ventilation according to Langeron et al[3] as "the inability of an unassisted anesthesiologist to maintain oxygen saturation $>92 \%$, as measured by pulse oximetry, or to prevent or reverse signs of inadequate ventilation during positive-pressure mask ventilation under general anesthesia".

Numerous previous studies on the prediction and treatment of DMV (difficult mask ventilation) concluded that Age $>55, \mathrm{BMl}>26 \mathrm{~kg} / \mathrm{m}^{2}$, presence of beard, edentulousness, and snoring are 5 independent factors related to DMV [3]. In addition, neck radiotherapy, neck circumference, male gender, Mallampti grade, and severely limited jaw protrusion are known as the risk factors for $\operatorname{DMV}[2,4,10]$. Among these studies, one of the independent factors of DMV is a history of snoring (Patients were asked if they were habitual snorers or not) [3]. Although OSA is very common[17], the majority of patients presenting to the operating room with OSA have not previously been diagnosed and lacked selfawareness of this condition. In this study, all patients underwent PSG preoperatively, which could provide detailed diagnoses of OSA and validate the accuracy of patient's self-reported history of snoring/choking for air during sleep. In conclusion, the PSG result was clinically more reliable than a declared history of snoring, as provided by the patients.

From a previous study, higher age, higher BMI, greater neck circumference, male gender, and higher Mallampati score were significantly correlated with a high risk of OSA[18]. Concurrently, these overlapping risk factors were also related to $\operatorname{DMV}[2-4,10]$. It was found that patients with OSA had a three to four-fold higher risk of difficult intubation or mask ventilation, or both, compared to non-sleep apnea patients[19]. However, most previous studies used diagnosed OSA as the primary outcome. While PSG is the diagnostic standard of OSA[11], one of its important parameters is $\mathrm{AHI}$, which is calculated by 
adding all apneas and hypopneas and then dividing by total sleep time. An AHI of 15 or more events per hour, or five or more events per hour in the presence of symptoms or cardiovascular comorbidities, is the diagnostic criteria for OSA[11, 12]. In this study, AHI was used as a new continuous measurement for DMV that provided more information the simple presence or absence of diagnosed OSA.

Normally, a conventional PSG examination is time-consuming and complicated to perform, requiring a minimum of 22 wires attached to the patient. With the development of home sleep testing devices, patients are more likely to accept and enjoy certain conveniences, as in this study. The portable PSG consisted of continuous recording with three sensors (respiratory effort belt, cannula and noninvasive pulse oximetry). Among the 200 patients, only 21 patients didn't have the PSG report due to inadequate time of measuring and feeling discomfort during the PSG testing. Concurrently, other parameters such as oxygen desaturation and heart rate during the night might further aid decision making for anesthesiologists.

As for the Chinese population, there was a limited number of articles on mask ventilation. Relatively, most of the trials were from the European and US populations. Furthermore, there were fewer bearded Chinese patients to consider, as well was differences in head and facial structures.

For the secondary outcome, age, $\mathrm{AHI}$ and Mallampati score were three significant, independent factors for the DMV. Most DMV evaluations consist of questionnaires which are time consuming for anesthesiologists to complete and score[20]. Additionally, it is unclear which predictive method is easier to perform and reliable for use in clinical settings. In our study, we demonstrated a possible solution that efficiently utilizes portable PSG, which, combined with AHI outputs, age, and Mallampati scores, can a predict patient's risk of DMV. Moreover, these three factors remained objective compared to the subjective answers extracted from questionnaires. Certain predictive models mutually benefit both the patient and anesthesiologist by saving time, avoiding recall bias, raising patient's satisfaction score, and providing a pre-liminary assessment of potential OSA in otherwise undiagnosed patients.

Once limitation of the study was the relatively small sample size $(n=159)$. To explore further applications of AHI in DMV patients, we will need to study larger patient populations. Also, our study focused on Chinese patients, which are ethnically different from other global populations. A larger, more versatile sample would be desirable for further study to demonstrate the effectiveness of our predictive model. Meanwhile, some of the patients reported an uncomfortable experience while they were sleeping during the PSG testing. Future models of PSG equipment should be developed to improve certain concerns from patient's perspective.

\section{Conclusion}

Elevated $\mathrm{AHI}$ is associated with increased risk of DMV. AHI, age, and Mallampti classification were independent factors of DMV in the Chinese population, and could altogether serve as a convenient and objective predictive model for DMV. 


\section{Declarations}

Acknowledgements

Jia-Yi Wang and Jing-Jie Li contributed equally as the first authors and were responsible for the clinical experiments and data analysis. Peng-Cheng Zhao, Jia-Li Peng, and Rong Hu contributed substantially to the data collections and interpretation. Xuan Pu contributed to the statistics analysis of the experiments. Hong Jiang took substantial responsibilities for the whole study and the writing of the manuscript.

\section{Funding}

This work is sponsored by Shanghai Municipal Science and Technology Commission (18441904600), Medical-Engineering Project of Shanghai Jiao Tong University (ZH2018ZDA14), and Clinical Project of Shanghai Ninth People's Hospital, Shanghai Jiao Tong University, School of Medicine (JYLJ040).

\section{Availability of data and materials}

The datasets generated and analyzed during the study are available from the corresponding author on reasonable request.

\section{Ethics approval and consent to participate}

This trial was approved by the ethics committee of Shanghai Ninth People's Hospital, Shanghai Jiao Tong University, School of Medicine. Written informed consent was obtained from all participants.

\section{Consent for publication}

Not applicable.

\section{Competing interests}

The authors declare no conflicts of interest in this work.

\section{References}

1. Apfelbaum JL, Hagberg CA, Caplan RA, Blitt CD, Connis RT, Nickinovich DG, Hagberg CA, Caplan RA, Benumof JL, Berry FA et al: Practice guidelines for management of the difficult airway: an updated report by the American Society of Anesthesiologists Task Force on Management of the Difficult Airway. Anesthesiology 2013, 118(2):251-270.

2. Shah PN, Sundaram V: Incidence and predictors of difficult mask ventilation and intubation. $J$ Anaesthesiol Clin Pharmacol 2012, 28(4):451-455.

3. Langeron O, Masso E, Huraux C, Guggiari M, Bianchi A, Coriat P, Riou B: Prediction of difficult mask ventilation. Anesthesiology 2000, 92(5):1229-1236. 
4. Kheterpal S, Martin L, Shanks AM, Tremper KK: Prediction and outcomes of impossible mask ventilation: a review of 50,000 anesthetics. Anesthesiology 2009, 110(4):891-897.

5. Crosby ET, Cooper RM, Douglas MJ, Doyle DJ, Hung OR, Labrecque P, Muir H, Murphy MF, Preston RP, Rose DK et al: The unanticipated difficult airway with recommendations for management. Can J Anaesth 1998, 45(8):757-776.

6. Caplan RA, Posner KL, Ward RJ, Cheney FW: Adverse respiratory events in anesthesia: a closed claims analysis. Anesthesiology 1990, 72(5):828-833.

7. Cook TM, Woodall N, Harper J, Benger J, Fourth National Audit P: Major complications of airway management in the UK: results of the Fourth National Audit Project of the Royal College of Anaesthetists and the Difficult Airway Society. Part 2: intensive care and emergency departments. $\mathrm{Br}$ $J$ Anaesth 2011, 106(5):632-642.

8. Law JA, Broemling N, Cooper RM, Drolet P, Duggan LV, Griesdale DE, Hung OR, Jones PM, Kovacs G, Massey $S$ et al: The difficult airway with recommendations for management-part 1-difficult tracheal intubation encountered in an unconscious/induced patient. Can J Anaesth 2013, 60(11):1089-1118.

9. Frerk C, Mitchell VS, McNarry AF, Mendonca C, Bhagrath R, Patel A, O'Sullivan EP, Woodall NM, Ahmad I, Difficult Airway Society intubation guidelines working g: Difficult Airway Society 2015 guidelines for management of unanticipated difficult intubation in adults. $\mathrm{Br} J$ Anaesth 2015, 115(6):827-848.

10. Yildiz TS, Solak M, Toker K: The incidence and risk factors of difficult mask ventilation. $J$ Anesth 2005, 19(1):7-11.

11. Kapur VK, Auckley DH, Chowdhuri S, Kuhlmann DC, Mehra R, Ramar K, Harrod CG: Clinical Practice Guideline for Diagnostic Testing for Adult Obstructive Sleep Apnea: An American Academy of Sleep Medicine Clinical Practice Guideline. J Clin Sleep Med 2017, 13(3):479-504.

12. Semelka M, Wilson J, Floyd R: Diagnosis and Treatment of Obstructive Sleep Apnea in Adults. Am Fam Physician 2016, 94(5):355-360.

13. Samsoon GL, Young JR: Difficult tracheal intubation: a retrospective study. Anaesthesia 1987, 42(5):487-490.

14. Lewis M, Keramati S, Benumof JL, Berry CC: What is the best way to determine oropharyngeal classification and mandibular space length to predict difficult laryngoscopy? Anesthesiology 1994, 81(1):69-75.

15. Smith S: Reporting the apnoea hypopnea index (AHI). J Clin Sleep Med 2007, 3(3):321.

16. Han R, Tremper KK, Kheterpal S, O'Reilly M: Grading scale for mask ventilation. Anesthesiology 2004, 101(1):267.

17. Young T, Peppard PE, Gottlieb DJ: Epidemiology of obstructive sleep apnea: a population health perspective. Am J Respir Crit Care Med 2002, 165(9):1217-1239.

18. Acar HV, Yarkan Uysal H, Kaya A, Ceyhan A, Dikmen B: Does the STOP-Bang, an obstructive sleep apnea screening tool, predict difficult intubation? Eur Rev Med Pharmacol Sci 2014, 18(13):18691874. 
19. Nagappa M, Wong DT, Cozowicz C, Ramachandran SK, Memtsoudis SG, Chung F: Is obstructive sleep apnea associated with difficult airway? Evidence from a systematic review and meta-analysis of prospective and retrospective cohort studies. PLoS One 2018, 13(10):e0204904.

20. Lundstrom LH, Rosenstock CV, Wetterslev J, Norskov AK: The DIFFMASK score for predicting difficult facemask ventilation: a cohort study of 46,804 patients. Anaesthesia 2019, 74(10):1267-1276.

\section{Figures}

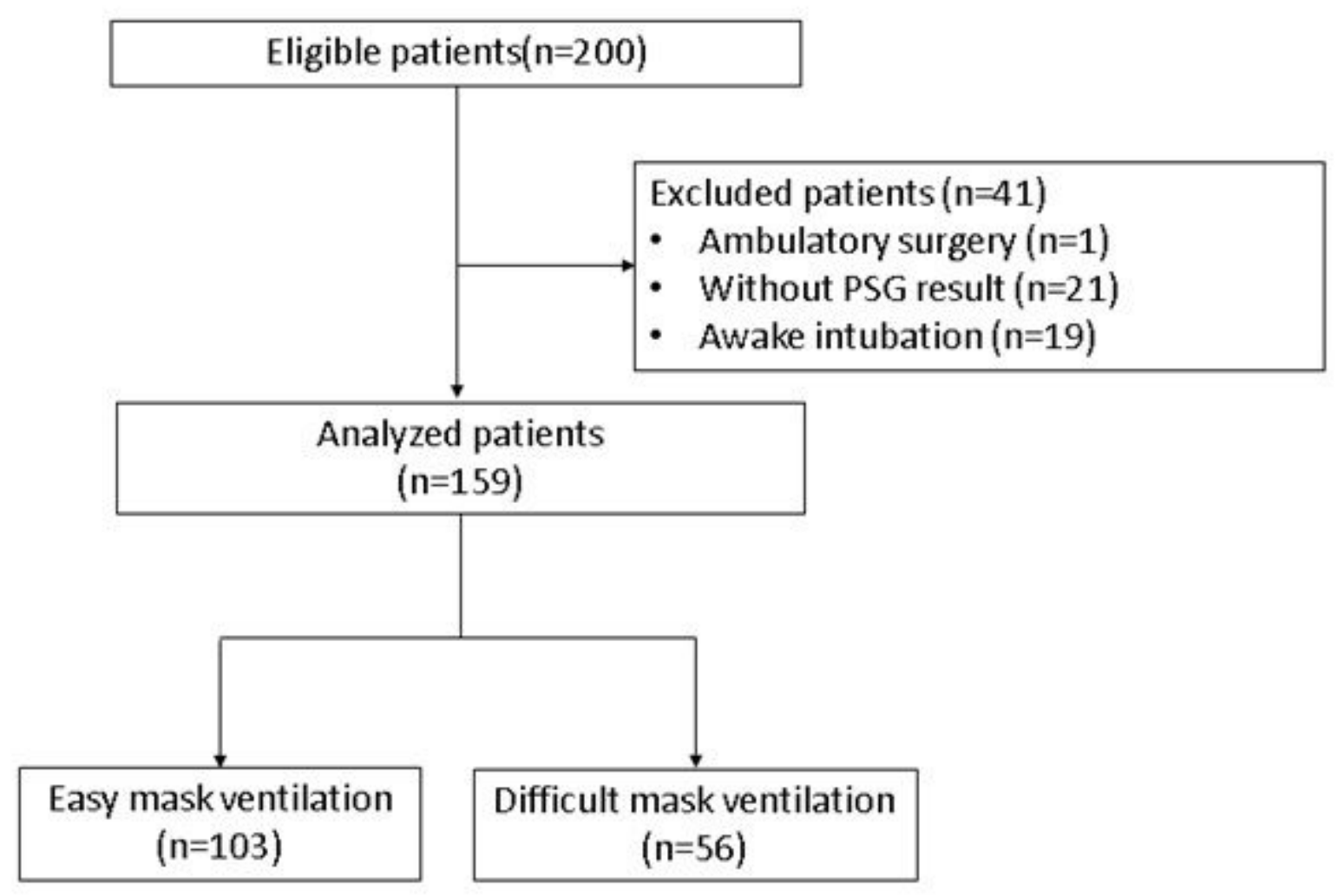

Figure 1

Patient enrollment flow diagram 


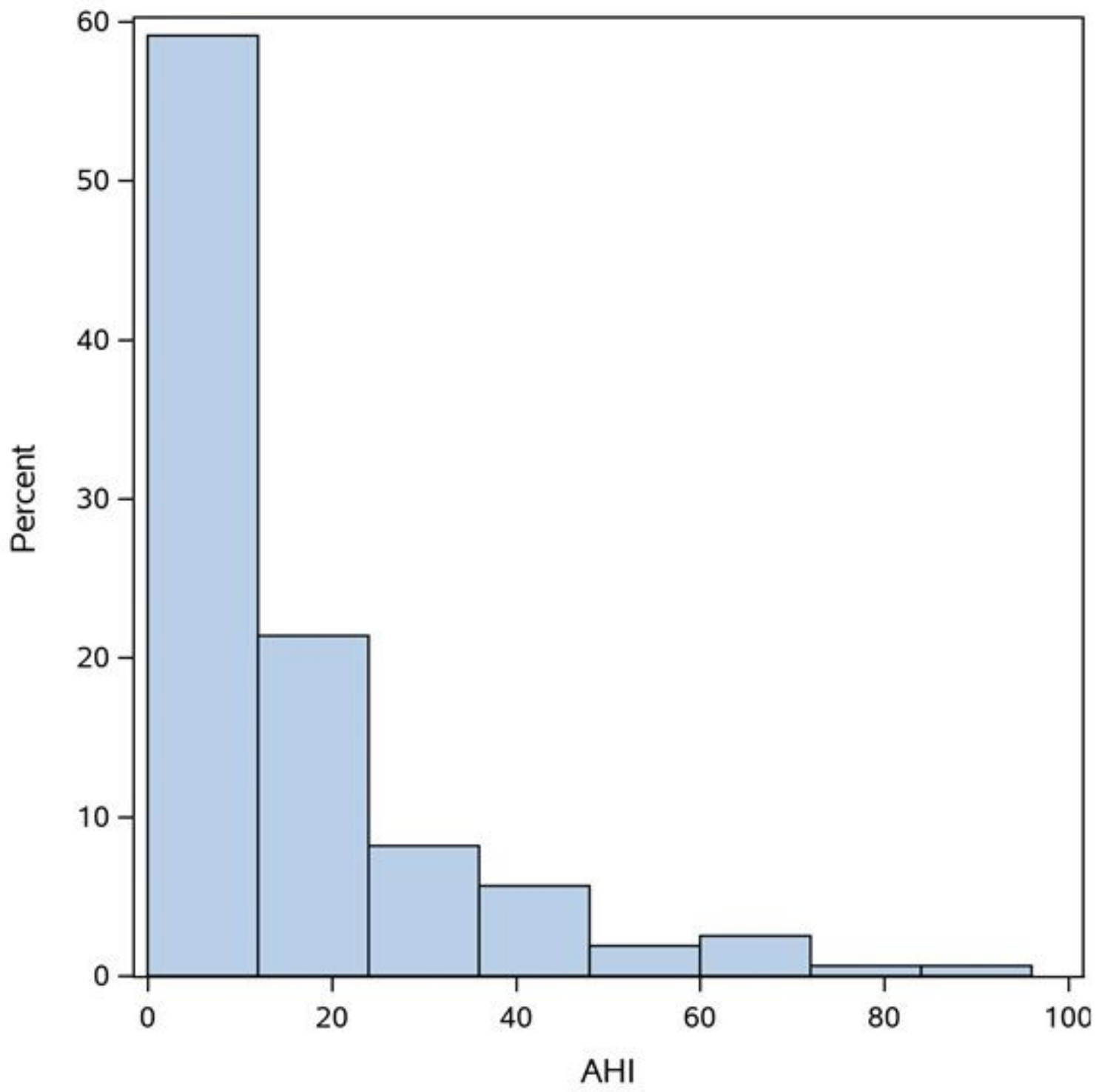

Figure 2

Histogram of Apnea-Hypopnea Index (AHI) 


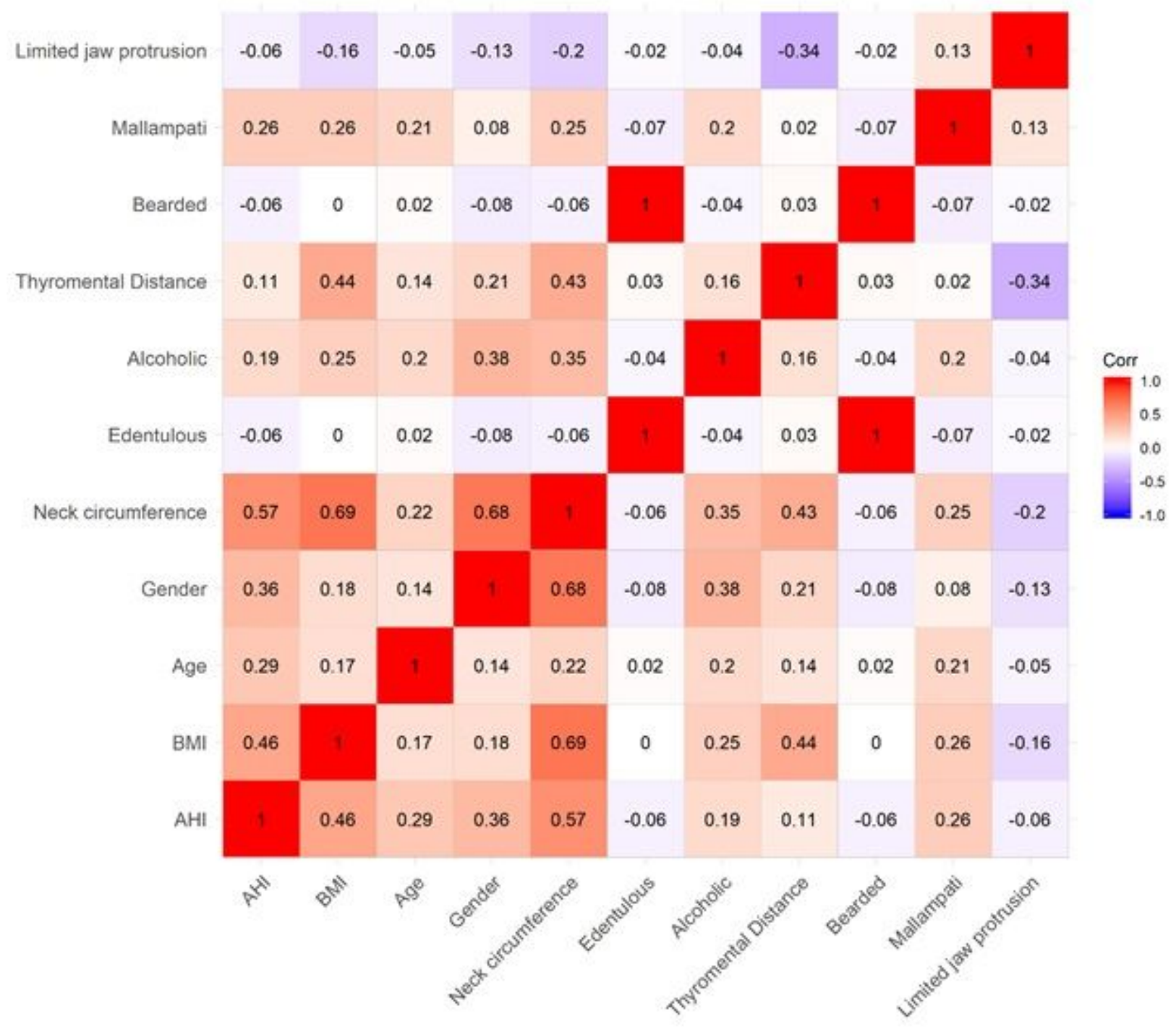

Figure 3

Pair-wise Pearson correlation coefficients between $\mathrm{AHI}$ and other variables, including age, gender, $\mathrm{BMI}$, neck circumference, modified Mallampti classification, thyromental distance, ability to extend lower jaw, and alcoholism 